\title{
Maternal schistosomiasis: IL-2, IL-10 and regulatory T lymphocytes to unrelated antigen in adult offspring mice
}

\author{
Erica de Souza Fernandes ${ }^{[1]}$, Virgínia Maria de Barros Lorena ${ }^{[2]}$, \\ Iana Rafaela Fernandes Sales ${ }^{[1]}$, Mônica Camelo Pessoa de Azevedo Albuquerque ${ }^{[1],[3],}$ \\ Yara de Miranda Gomes ${ }^{[2]}$, Vlaudia Maria Assis Costa ${ }^{[1],[3]}$ \\ and Valdênia Maria Oliveira de Souza ${ }^{[1],[4]}$
}

\begin{abstract}
[1].Laboratório de Imunopatologia Keizo Asami, Universidade Federal de Pernambuco, Recife, PE, Brasil.
[2]. Instituto Aggeu Magalhães, Laboratório de Imunologia, Fundação Oswaldo Cruz, Recife, PE, Brasil.

[3]. Departamento de Medicina Tropical, Centro de Ciências da Saúde, Universidade Federal de Pernambuco, Recife, PE, Brasil.

[4]. Departamento de Ciências Farmacêuticas, Centro de Ciências da Saúde, Universidade Federal de Pernambuco, Recife, PE, Brasil.
\end{abstract}

\begin{abstract}
Introduction: We evaluated IL-10, IL-2 and regulatory T cells (Treg), in response to ovalbumin (OA), in offspring from schistosomotic mouse mothers. Methods: We used animals born (BIM) or suckled (SIM) from infected mothers; and mice born/suckled from infected (BSIM) or non-infected mothers (CONTROL). After OA+adjuvant immunization, spleen cells were cultured, with or without OA, and doubly marked for cytometry. Results: BIM showed fewer CD4+/IL-2+ and more B220+/IL-10+ cells, whereas the SIM group showed increased Treg frequency. BSIM had fewer B220+/IL-10+ and Treg cells. Conclusions: Separately, gestation or nursing induced immunosuppressive cells in infected mothers, but improved anti-OA immunity when combined.
\end{abstract}

Keywords: Maternal schistosomiasis. Adult offspring. Immunomodulation.

Schistosoma mansoni infection is highly prevalent in tropical regions, including Northeast and Southeast Brazil ${ }^{1}$. In endemic regions, there is a high prevalence of Schistosoma infections in pregnant women ${ }^{2}$. During the chronic phase of schistosomiasis, egg antigens induce T-helper 2 (Th2)dependent granuloma [interleukin (IL)-4, IL-13, IL-5, and eosinophils], which negatively regulate the Th1 response [interferon gamma (IFN- $\gamma$ ), tumor necrosis factor alpha (TNF- $\alpha$ ), and macrophages], triggered by infectious larvae or heterologous antigens ${ }^{3}$. IL-10, Treg and regulatory B (Breg) cells down-regulate both the Th1 and Th2 immune responses during the chronic phase $e^{4,5}$.

The attenuation of the granulomatous inflammatory response was also observed in the offspring of infected mothers ${ }^{6}$. This immunomodulatory phenomenon of homologous antigen was associated with the transfer of parasite antigens or anti-parasite antibodies through the placenta or breast milk ${ }^{7}$. However, very little is known about the alterations of the immune response to heterologous antigens in the offspring of schistosomotic mothers ${ }^{8,9}$.

Corresponding author: $\mathrm{Dr}^{\mathrm{a}}$ Valdênia Maria Oliveira de Souza.

e-mail: valdenia.souza@gmail.com

Received 19 December 2016

Accepted 28 February 2018
In an experimental model, this phenomenon was shown to be related to the effects of pregnancy or nursing in infected mothers on the immune response to ovalbumin (OA). Suckling from infected mothers enhanced the OA-specific humoral response and IL-2 secretion. In contrast, gestation in S. mansoni-infected mice impaired OA-specific humoral immunity in an IL-10dependent manner ${ }^{10}$. Here, the production of IL-10 and IL-2 in immune cells was investigated, as well as the frequency of the Treg cells, in response to OA in these offspring.

Four-week-old, female Swiss Webster mice were infected with 20 S. mansoni cercariae, São Lourenço da Mata (SLM) strain. At 60 days post-infection, the females were caged with male mice at a 1:1 ratio. The same procedure was performed with non-infected females. Immediately after birth, the newborns from S. mansoni-infected or non-infected mothers were housed in cages with interchanged mothers. After adoptive nursing, four groups of male mice were formed $(n=10)$ : offspring mice born from infected mothers (BIM) suckling from noninfected mothers; offspring of non-infected mothers suckling from infected mothers (SIM); and two groups of mice born and suckling from infected mothers (BSIM) or non-infected (CONTROL) mothers. Six-week-old mice were immunized, subcutaneously, with $100 \mu$ g of ovalbumin (OA) (grade V; Sigma-Aldrich) emulsified in complete Freund's adjuvant (CFA) (Sigma-Aldrich), at the base of the tail $(0.1 \mathrm{ml} /$ animal). 
The mice were housed in the animal care facility at the Aggeu Magalhães Institute, Oswaldo Cruz Foundation-FIOCRUZ, Recife, PE, Brazil.

After 8 days of immunization, the spleen cell suspension was aseptically obtained and cultivated (humidified incubator $\mathrm{CO}_{2}$; at $37^{\circ} \mathrm{C}$ ) at a final concentration of $5 \times 10^{6}$ cells $/ \mathrm{ml}$ with OA $(500 \mu \mathrm{g} / \mathrm{ml})$ or without antigenic stimulus, or in only culture medium (non-stimulated cells), for $24 \mathrm{~h}$. Double labeling was performed after incubation $\left(4^{\circ} \mathrm{C}, 30 \mathrm{~min}\right)$ with fluorochrome-labeled antibody solutions, at a concentration of $0.5 \mathrm{mg} / 10^{6}$ cells: PE-Cy 5 (phycoerythrin and cyanine) anti-mouse cluster of differentiation (CD)3, fluoresceína isotiocianato (FITC) anti-mouse B220, or FITC anti-mouse CD14 plus PE anti-mouse IL-10; FITC anti-mouse CD4 and Peridinin-Chlorophyll-protein (PerCP) anti-mouse IL-2 or PE anti-mouse FoxP3 (BD Biosciences). After washing, the cells were analyzed by flow cytometry (FACSCalibur, BD-Pharmingen, San Diego, CA, USA). The frequency of positive cells was analyzed using the program Cell Quest: The lymphocyte region was determined using a granularity SSC (Side Scatter) and size FSC (Forward Scatter) plot. Antigen-presenting cells were selected based on granularity and expression of CD14+ or B220+. Limits for the quadrant markers were always set based on negative populations and isotype controls. A descriptive analysis of the frequency of cells in the upper right quadrant (double-positive cells) was performed. Statistical differences $(p<0.05)$ between groups were assessed using a one-way analysis of variance and Mann-Whitney test. All procedures were repeated three times.

After 8 days of OA in vivo immunization, the non-stimulated cell cultures from the offspring born from schistosomotic mothers showed significantly lower CD4+/IL-2+ cell frequency $(\mathrm{SIM}=0.3 \% ; \mathrm{BIM}=0.1 \% ; \mathrm{BSIM}=0.1 \% ; \mathrm{CONTROL}=0.4 \%)$.
Upon in vitro OA stimulation, low CD4+/IL-2+ frequency was observed in the SIM group when compared to CONTROL and other experimental groups ( $\mathrm{SIM}=0.2 \%$; $\mathrm{BSIM}=0.5 \%$; $\mathrm{BIM}=0.8 \%$; CONTROL $=0.8 \%)($ Figure $1 \mathrm{~A})$. With regards to CD4+/FoxP3+ cells, we observed high frequency in the SIM group, without in vitro antigenic stimulus, when compared to that in the CONTROL group ( $\mathrm{SIM}=1.5 \%$; $\mathrm{BSIM}=0.6 \%$; $\mathrm{BIM}=0.6 \%$; $\mathrm{CONTROL}=0.4 \%$ ) (Figure 1B).

With regards to CD3+ (Figure 2A), B220+ (Figure 2B), $\mathrm{CD} 14+$ (Figure 2C) cells and IL-10 production, there was a significantly increased B220+/IL-10+ cell frequency in the BIM group when stimulated with OA, in comparison to that of non-stimulated cells (Figure 2B).

Based on these results, pregnancy affords a decrease in intracellular IL-2 production by $\mathrm{T}$ lymphocytes and favors the presence of B lymphocytes with IL-10. These finding suggest the existence of an immune-suppressive background, that may be due to the passage, through the placenta, of antigens from eggs and worms of S. mansoni, inducing the generation of IL-10producing dendritic cells and $\mathrm{B}$ lymphocytes ${ }^{11,12}$. It is important to mention that after in vitro OA restimulation, IL-2 production was recovered and a higher frequency of IL-10-producing B lymphocytes was achieved. Taken together, these results suggest partial impairment of the anti-OA immune response in BIM mice. In fact, we observed notable damage to the humoral response (antibodies production) and a slight alteration in cellular immunity in response to OA in these offspring ${ }^{10}$.

Previous contact with milk from infected mothers improved Treg cell frequency after in vivo OA immunization. It is possible that the parasitic antigen properties in eliciting Treg cell responses ${ }^{4}$, together with the suppressive microenvironment of the gut mucosa $^{13}$, enabled this Treg background. Nevertheless, the OA restimulation in vitro lowered the Treg levels (Figure 1B, white bar).

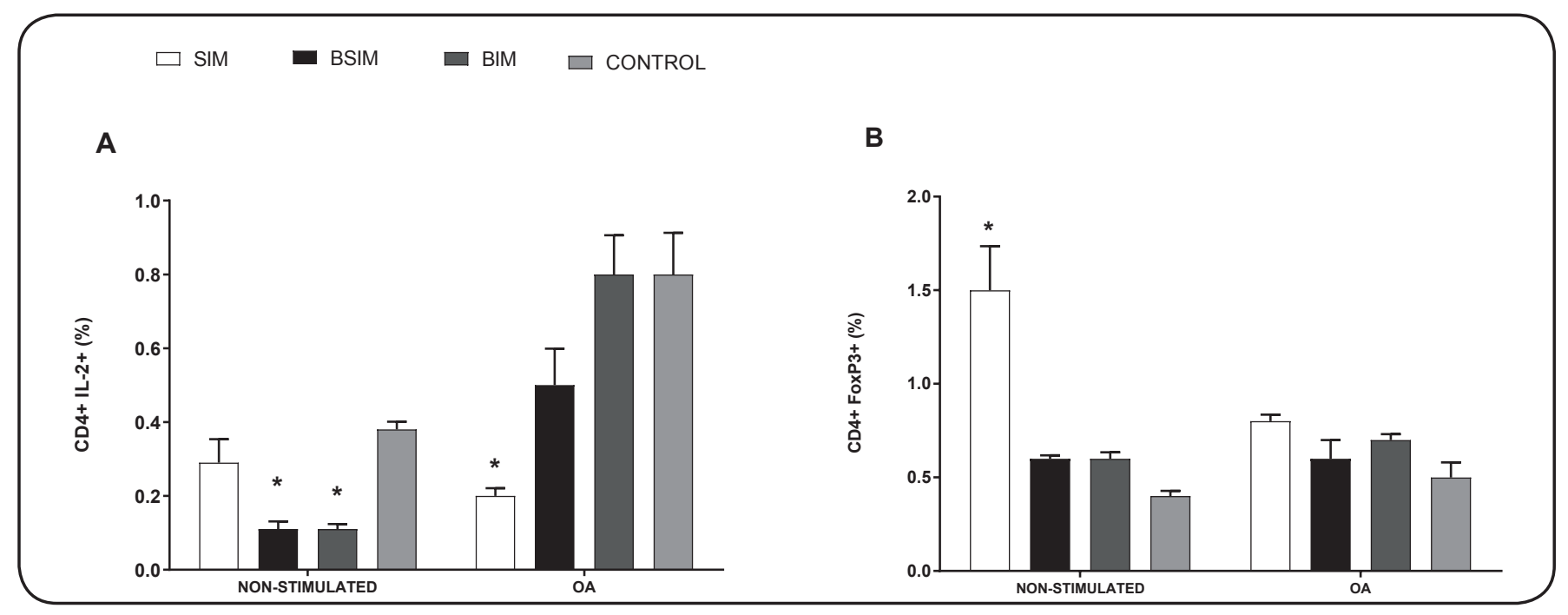

FIGURE 1: Frequency of CD4+ and IL-2+(A) or FoxP3+(B) cells in adult mice born (BIM) or suckling in infected mothers (SIM); mice born and suckling

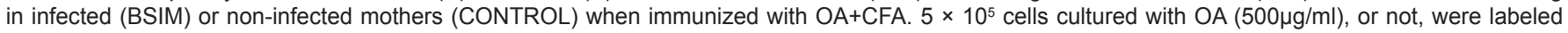
with FITC anti-CD4 and PerCP anti-IL2 (A) or PE anti-FoxP3 (B). SIM: suckling in infected mothers; BSIM: born and suckling from infected mothers; BIM: born from infected mothers; CONTROL: non-infected mothers; OA: ovalbumin; CD4+: cluster of differentiation 4+; IL: interleukin; CFA: complete Freund's adjuvant; FITC: fluoresceína isotiocianato; PE: phycoerythrin. * $p<0.05$ when compared to respective CONTROL groups. 


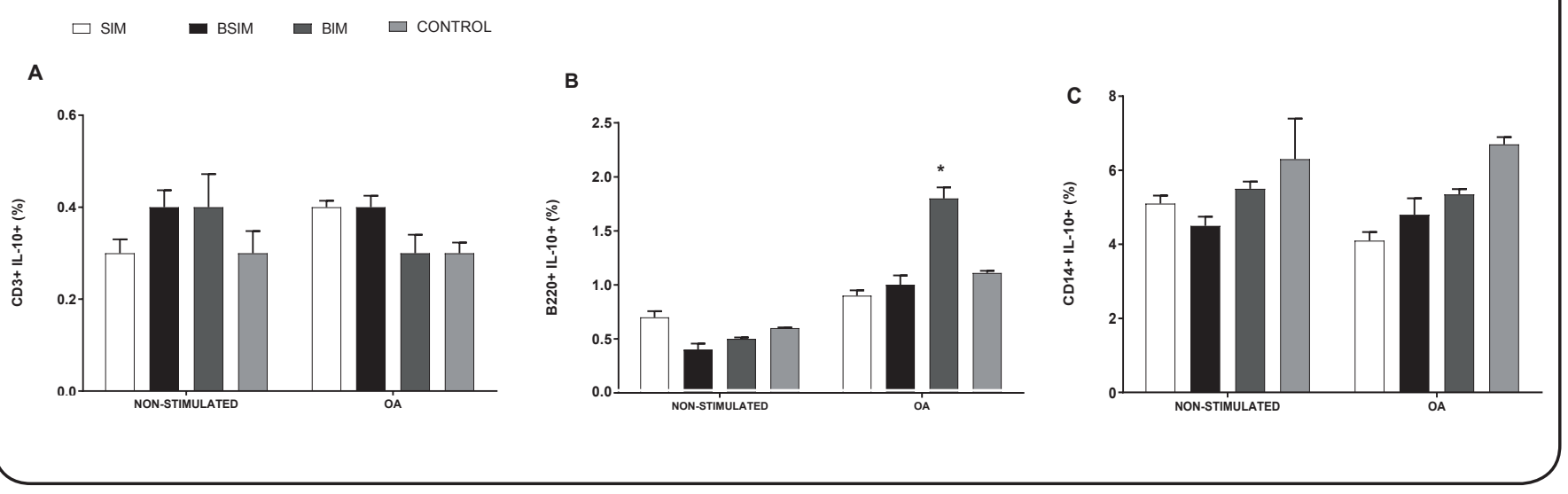

FIGURE 2: Frequency of CD3+(A), B220+(B), or CD14+(C) cells, and IL-10+ in adult mice born (BIM) or suckling in infected mothers (SIM); mice born and

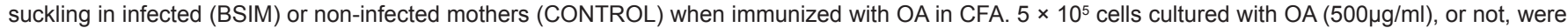
labeled with PE-Cy5 anti-CD3(A), FITC anti-B220(B), or FITC anti-CD14(C), and PE anti-IL-10. SIM: suckling in infected mothers; BSIM: born and suckling from infected mothers; BIM: born from infected mothers; CONTROL: non-infected mothers; CD: cluster of differentiation; IL: interleukin; OA: ovalbumin; CFA: complete Freund's adjuvant; FITC: fluoresceína isotiocianato; PE: phycoerythrin. ${ }^{*} \mathrm{p}<0.05$ when compared to non-stimulated cells from BIM groups.

These results suggest that immunization with CFA followed by a boost antigen (for example, in vitro restimulation) can render Treg-mediated immune regulation defective. APC-derived cytokines and intracellular signal transduction pathways induce an anti-OA stimulatory environment that can hinder Treg cell activation ${ }^{14}$. Interestingly, intracellular IL-2 production by lymphocytes in response to OA was not observed. This could be due to its rapid secretion after in vitro antigenic stimulus. In agreement with this, high levels of this cytokine were detected in the supernatant from the SIM group cell culture ${ }^{10}$.

Interestingly, the BSIM group had attenuated immunosuppressive cells compared to the SIM and BSIM groups: few Treg cells plus the recovery of IL-2 production and low induction of B220+IL-10+ cells in response to OA. Our results therefore highlight the importance of pregnancy followed by nursing for controlling immunosuppressive status of offspring during their adult life.

The expansion of Breg and Treg cell types (IL-10/IFN- $\gamma$ double producers and Foxp3+ Tregs) might contribute to prevention of diabetes in Non Obese Diabetic (NOD) or asthmatic mice $^{5,15}$. Previously, $S$. mansoni antigens were able to inhibit the Th1-mediated autoimmune disease Type 1 diabetes ${ }^{15}$, as well as Th2-meditated allergic diseases. Therefore, the demonstration of resistance or susceptibility of offspring during the development of allergic disorders that are inherently Th2-driven, as compared with Inflammatory Bowel Disease (IBD), in which the pathophysiology is mediated by Th1/Th17 responses, would be important to identify the regulatory mechanism in offspring from schistosomotic mothers.

These results suggest that contact with parasitic antigens in early life can modulate immunity to non-related antigens, such as those of autoimmune diseases, allergies, heterologous infections, and vaccines in individuals from areas endemic for schistosomiasis.

\section{Ethical considerations}

The experimental protocol was approved by the Ethics Committee for Animal Research (CEUA) of the Aggeu Magalhães Institute/Oswaldo Cruz Foundation (IAM/FIOCRUZ) (number protocol: 25/2011).

\section{Acknowledgment}

We would like to thank veterinary Gerlane Chioratto for competent assistance.

\section{Conflict of interest}

The authors declare that there is no conflict of interest.

\section{Financial support}

Coordenação de Aperfeiçoamento de Pessoal de Ensino Superior (CAPES), master's and doctorates fellowship.

\section{REFERENCES}

1. World Health Organization (WHO). Schistosomiasis, 2011. Available from: http://www.who.int/tdr/diseases-topics/schistosomiasis/en/

2. Friedman JF, Mital P, Kanzaria HK, Olds GR, Kurtis JD. Schistosomiasis and pregnancy. Trends Parasitol. 2007;23(4):159-64.

3. Caldas IR, Campi-Azevedo AC, Oliveira LFA, Silveira AMS, Oliveira RC, Gazzinelli G. Human schistosomiasis mansoni: immune responses during acute and chronic phases of the infection. Acta Trop. 2008;108(2-3):109-17.

4. El-Ahwany E, Bauiomy IR, Nagy F, Zalat R, Mahmoud O, Zada S. T Regulatory cell responses to immunization with a soluble egg antigen in Schistosoma mansoni-infected mice. Korean J Parasitol. 2012;50(1):29-35.

5. Van der Vlugt LEPM, Labuda LA, Ozir-Fazalalikhan A, Lievers E, Gloudemans AK, Liu KY, et al. Schistosomes induce regulatory 
features in human and mouse CD1d(hi) B cells: inhibition of allergic inflammation by IL-10 and regulatory T cells. PLoS One. 2012;7(2):e30883.

6. Othman AA, Shoheib ZS, Saied EM, Soliman RH. Congenital exposure to Schistosoma mansoni infection: impact on the future immune response and the disease outcome. Immunobiology. 2010;215(2):101-12.

7. Montesano MA, Colley DG, Freeman GL, Secor WE. Neonatal exposure to idiotype induces Schistosoma mansoni egg antigenspecific cellular and humoral immune responses. J Immunol. 1999;163(2):898-905.

8. Noureldin MS, Shaltout AA. Anti-schistosomal IgE and its relation to gastrointestinal allergy in breast-fed infants of Schistosoma mansoni infected mothers. J Egypt Soc Parasitol. 1998;28(2):539-50.

9. Malhotra I, Mungai P, Wamachi A, Kioko J, Ouma JH, Kazura JW, et al. Helminth- and Bacillus Calmette-Guérin-induced immunity in children sensitized in utero to filariasis and schistosomiasis. J Immunol. 1999;162(11):6843-8.
10. Santos PEA, Sales IRF, Schirato GV, Costa VM, Albuquerque MC, Souza VM, et al. Influence of maternal schistosomiasis on the immunity of adult offspring mice. Parasitol Res. 2010;107(1): 95-102.

11. Thomas PG, Harn Jr DA. Immune biasing by helminth glycans. Cell Microbiol. 2004;6(1):13-22.

12. Harris N, Gause WC. B cell function in the immune response to helminths. Trends Immunol. 2011;32(2):80-8.

13. Weiner HL, da Cunha AP, Quintana F, Wu H. Oral tolerance. Immunol Rev. 2011;241(1):241-59.

14. Riha P, Rudd CE. CD28 co-signaling in the adaptive immune response. Self Nonself. 2010;1(3):231-40.

15. Zaccone P, Burton OT, Gibbs S, Miller N, Jones FM, Dunne DW, et al. Immune modulation by Schistosoma mansoni antigens in NOD mice: effects on both innate and adaptive immune systems. J Biomed Biotechnol. 2010;2010:1-11. ID: 795210. 